Article

\title{
Generation Mechanism of Nonlinear Rayleigh Surface Waves for Randomly Distributed Surface Micro-Cracks
}

\author{
Xiangyan Ding ${ }^{1}$, Feilong $\mathrm{Li}^{1}$, Youxuan Zhao ${ }^{1, *(\mathbb{D})}$, Yongmei $\mathrm{Xu}^{1}$, Ning $\mathrm{Hu}^{1,2, * \mathbb{C}}$, Peng Cao ${ }^{3}$ \\ and Mingxi Deng ${ }^{1}$ \\ 1 College of Aerospace Engineering, Chongqing University, Chongqing 400044, China; \\ ddingxiangyan@yeah.net (X.D.); feitian2302@sina.com (F.L.); aht2010@sina.com (Y.X.); \\ dengmx65@yahoo.com (M.D.) \\ 2 Key Laboratory of Optoelectronic Technology and Systems of the Education Ministry of China, \\ Chongqing University, Chongqing 400044, China \\ 3 Department of Hydraulic Engineering, Tsinghua University, Beijing 100084, China; caopeng518888@126.com \\ * Correspondence: youxuan.zhao@cqu.edu.cn (Y.Z.); ninghu@cqu.edu.cn (N.H.)
}

Received: 11 April 2018; Accepted: 19 April 2018; Published: 23 April 2018

\begin{abstract}
This paper investigates the propagation of Rayleigh surface waves in structures with randomly distributed surface micro-cracks using numerical simulations. The results revealed a significant ultrasonic nonlinear effect caused by the surface micro-cracks, which is mainly represented by a second harmonic with even more distinct third/quadruple harmonics. Based on statistical analysis from the numerous results of random micro-crack models, it is clearly found that the acoustic nonlinear parameter increases linearly with micro-crack density, the proportion of surface cracks, the size of micro-crack zone, and the excitation frequency. This study theoretically reveals that nonlinear Rayleigh surface waves are feasible for use in quantitatively identifying the physical characteristics of surface micro-cracks in structures.
\end{abstract}

Keywords: nonlinearity; Rayleigh surface waves; micro-cracks; numerical simulation

\section{Introduction}

Stress corrosion micro-cracks, on or near a material surface, can susceptibly occur in metallic structures due to fatigue loads and corrosive environments [1,2]. Thus, it is of crucial importance to develop various non-destructive testing methods to detect and evaluate surface cracks for ensuring the integrity and safety of metallic structures. Unfortunately, it is well-known that linear ultrasonic methods based non-destructive testing technologies are not capable of detecting closed micro-cracks $[3,4]$. On the other hand, many studies have demonstrated that nonlinear ultrasonic-based non-destructive testing technologies are effective for evaluating early material degradation and micro-crack initiation by analyzing the nonlinear effects of ultrasonic waves [5-12].

The energy of Rayleigh surface waves is generally concentrated in the region near the surface within a depth of about one wavelength, and therefore, it is convenient and essential to utilize them to detect and evaluate surface cracks. In recent years, great efforts have been devoted to the detection of early material degradation using the higher harmonics of Rayleigh surface waves [13-21]. For instance, Achenbach et al. [13] have conducted an analytical far-field solution for the cumulative third harmonic surface wave, propagating on a half-space of isotropic incompressible cubically nonlinear material, in a relatively simple and systematic manner. Herrmann et al. [14] have reported a linear increase of the amplitude of a second harmonic surface wave using propagation distance by an experimental investigation. 
It is supposed that when elastic waves reach a micro-crack, the crack can be closed and opened under compression and tension [22-24], and the compressive part of Rayleigh waves can penetrate through the crack while the tensile part cannot. The higher harmonics are generated due to the apparent local stiffness changes at the crack region under tension and compression. However, compared with works on early material degradation, research on the detection of micro-cracks using higher harmonics of Rayleigh surface waves is still rarely reported [6]. Oberhardt et al. [6] have modelled randomly distributed surface-breaking micro-cracks and investigated their relationship to second harmonic generation in Rayleigh surface waves with micro-crack density. In their study [6], a hyperelastic constitutive law was derived and implemented to simplify the micro-crack model. Rjelka et al. [25] have determined the relationship between material properties (third-order elastic constants) and inhomogeneous distributions of micro-cracks. They then investigated the acoustic nonlinearity parameter for surface acoustic waves based on second harmonic generation.

In this work, and different from Oberhardt et al. [6] and Rjelka et al. [25], the simplified micro-crack model is not used. We employ a true contact model for modelling the micro-cracks. Besides considering the normal contact stiffness, the Coulomb friction law for the tangential direction is adopted in the contact model, and the linear elastic law for material is employed. We focus on the mechanism of second harmonic generation and propagation of Rayleigh surface waves in metallic structures with randomly distributed surface micro-cracks. In addition, the quantitative relationships between the acoustic nonlinear parameter and the micro-cracks are also studied. This work will provide a theoretical basis for detection and evaluation techniques based on nonlinear Rayleigh surface waves.

\section{Acoustic Nonlinearity Parameter for Rayleigh Waves}

To date, a substantial number of experiments have demonstrated that the nonlinear ultrasonic detection technique, based on the second harmonics of bulk waves, Lamb waves, and Rayleigh surface waves, is effective for detecting changes to the microstructure of metal materials [26]. The amplitude of the second harmonic waves can be quantitatively evaluated using the acoustic nonlinearity parameter.

Considered a weak quadratic nonlinearity, the acoustic nonlinearity parameter of Rayleigh surface waves at the surface $\overline{u_{y}}=u_{y}(y=0)$ can be expressed as $[14,18,26]$ :

$$
\beta=\frac{\overline{u_{y}}(2 \omega)}{\overline{u_{y}^{2}(\omega) x}} \frac{i 8 p}{\kappa_{P}^{2} \kappa_{R}}\left(1-\frac{2 \kappa_{R}^{2}}{\kappa_{R}^{2}+s^{2}}\right)
$$

where $p^{2}=\kappa_{R}^{2}-\kappa_{P}^{2}, s^{2}=\kappa_{R}^{2}-\kappa_{S}^{2}, \kappa_{R}, \kappa_{P}$, and $\kappa_{S}$ are the wavenumbers for the Rayleigh, longitudinal, and shear waves, respectively.

However, the contact acoustic nonlinearity caused by cracks is different from classical acoustic nonlinearity. Therefore, a different acoustic nonlinearity parameter $\beta^{\prime}=A_{2} / A_{1}$ is used in this study [22-24], where $A_{1}$ and $A_{2}$ are, respectively, the amplitudes of the fundamental and second harmonic waves. This parameter is related to the crack density, the frequency of fundamental waves, the propagation distance, and the friction coefficient.

\section{Numerical Simulation}

Since it is a challenge to directly obtain the nonlinear response when a Rayleigh wave passes through micro-cracks by an analytic method, numerical simulations are employed here. A two-dimensional finite element method (FEM) model of a structure with randomly distributed micro-cracks is built using the commercial FEM software ABAQUS (Version 6.14) and Python (Version 2.7).

The problem of Rayleigh surface waves propagating through a structure with surface micro-cracks is described in Figure 1. The micro-cracks are at or near the surface of the middle region of the specimen, which is named as a micro-crack zone with length $L$ (Note that the range of $L$ in this paper is from $10 \mathrm{~mm}$ to $30 \mathrm{~mm}$ ). In order to excite Rayleigh surface waves, longitudinal waves are applied using a dynamic displacement excitation on the flank of the wedge. Rayleigh waves are then excited due to 
the Rayleigh critical angle $\theta$. These waves propagate along the positive direction of $x$, and interact with the micro-cracks when they reach the micro-crack zone. As a result, the second harmonic waves are generated. Because the size of micro-cracks is much smaller than the wavelength of Rayleigh surface waves, most fundamental waves pass through the micro-crack zone and are collected at three signal detection positions (named as transmitted waves), which are $0 \mathrm{~mm}, 40 \mathrm{~mm}$, and $80 \mathrm{~mm}$ away from the right boundary of the micro-crack zone, respectively, as shown in Figure 1.

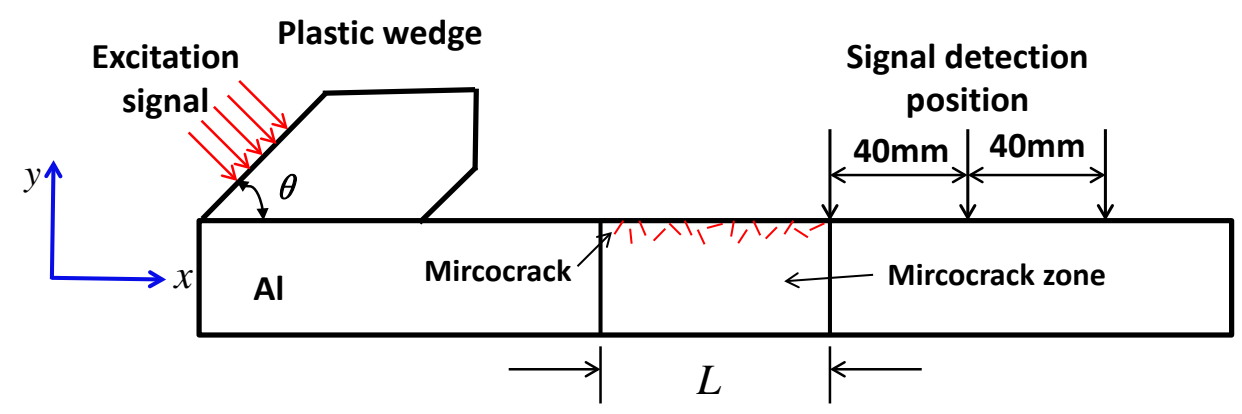

Figure 1. Schematic of Rayleigh surface waves propagating through a region with micro-cracks.

To investigate the influence of micro-cracks on the acoustic nonlinearity parameter, $N$ micro-cracks are randomly distributed at or near the surface of the middle part with length $L$. Note that when micro-cracks are near the surface, the distances from the surface to the centers of micro-cracks are less than the wavelength of the Rayleigh wave. The parameter $w \%$ is defined as the ratio of the number of surface micro-cracks divided by the total number of micro-cracks, which represents the proportion of micro-cracks on the surface. Thus, $w \%=100 \%$ means that all the micro-cracks are on the surface. Additionally, all the cracks have the same length of $2 a$ (the range of $a$ is from $25 \mu \mathrm{m}$ to $75 \mu \mathrm{m}$ in this study). Therefore, the crack density can be defined as $c=N a / L$, where $c$ is a dimensionless parameter. In this study, there are two variables that affect the random distribution of micro-cracks, i.e., the center position and the orientation of cracks, which are described by the probability density function (PDF) with a uniform random variable in the simulations.

A linear elastic constitutive law of aluminum (AL-6061-T6) for the specimen and plastics for the wedge are considered in the FEM analysis, and the material properties used in the simulations are $\rho_{\mathrm{Al}}=2704 \mathrm{~kg} / \mathrm{m}^{3}, E_{\mathrm{Al}}=68.9 \mathrm{GPa}, v_{\mathrm{Al}}=0.33, \rho_{\text {Plastic }}=1180 \mathrm{~kg} / \mathrm{m}^{3}, E_{\text {Plastic }}=4 \mathrm{Gpa}$, and $v_{\text {Plastic }}=0.33$. Thus, the Rayleigh critical angle $\theta=53.28^{\circ}$ can be calculated by the material properties of aluminum and plastics.

The size of the FEM model is $240 \times 6 \mathrm{~mm}$. Since at least 20 elements are required in the wavelength of the highest excitation frequency waves $(2000 \mathrm{kHz}$ in this study), from the aspect of numerical accuracy, at least 6 elements for each crack are employed with certainty, and the element size for the non-crack zone is set to $L_{\max }=0.08 \mathrm{~mm}$. The FEM model is constructed using the four-node plane strain (CPE4R) elements, as shown in Figure 2.

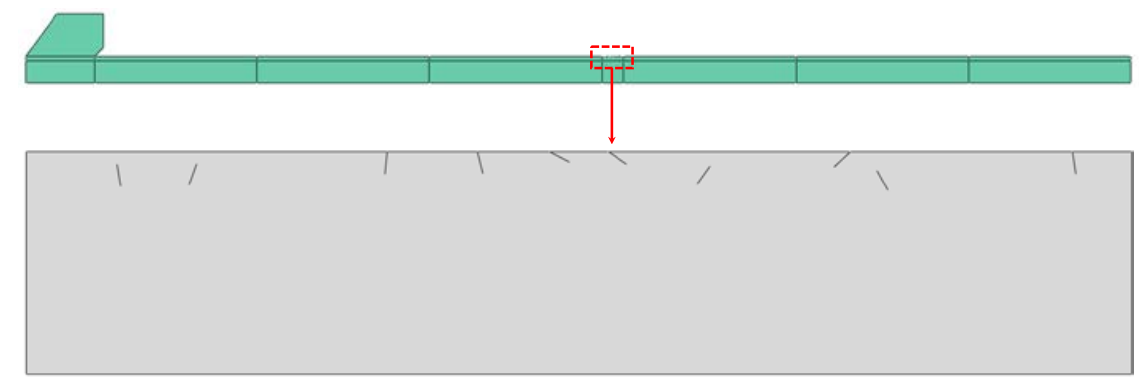

(a)

Figure 2. Cont. 


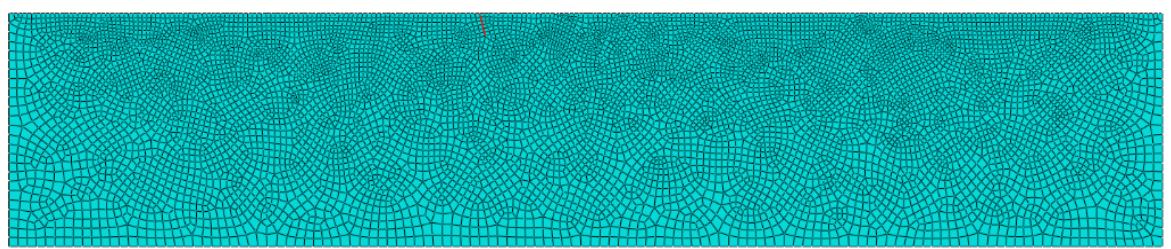

(b)

Figure 2. FEM model of the micro-crack zone: (a) distribution of micro-cracks; (b) finite element mesh (the red line represents one crack in the micro-crack zone).

A micro-crack is modelled as two contact surfaces, which can be separated from, but cannot interpenetrate into, each other. In addition, the Coulomb friction law is employed to model the relative sliding of the surfaces, and the friction coefficient is $\mu$. Thus, the actual traction applied to the crack faces is given by [8]:

$$
\mathbf{q}=\sigma_{0} \mathbf{n}+\left[\tau_{0}+\mu \sigma_{0} H\left(-\sigma_{0}\right)\right] H\left[\tau_{0}+\mu \sigma_{0} H\left(-\sigma_{0}\right)\right] \mathbf{s}
$$

where $\sigma_{0}$ and $\tau_{0}$ are, respectively, the normal and shear stresses on the surfaces of the crack, $H$ is the Heaviside step function, $\mathbf{n}$ is a unit vector that is perpendicular to the crack face, and $\mathbf{s}$ is a unit vector in the plane of the crack face.

The dynamic displacement excitation applied to the flank of the wedge can be expressed as $u(x, t)=A_{0} \sin (2 \pi f t) \times \sin (\pi f t / 10)^{2}$, where $A_{0}$ is the amplitude of excitation signal $\left(1 \times 10^{-4} \mathrm{~mm}\right.$ in this study $[8,27,28]$ ) and $f$ is the frequency of the excitation signal (ranging from $800 \sim 2000 \mathrm{kHz}$ ). In addition, the frequency needs to be low enough to satisfy the low frequency assumption, leading to $\lambda / a \approx 20$, where $\lambda$ is the wavelength and $a$ is the half-crack length [24]. Furthermore, the non-reflecting or absorbing boundary conditions at the bottom and right edges of the FEM model are used to maximally eliminate the influence of boundary reflection.

In this study, a ABAQUS/Explicit solver, based on the central different method, is employed to simulate the propagation of Rayleigh surface waves in the time domain, which is conditionally stable. The smallest time increment for the current problem that leads to a stable solution is $\Delta t=1 \times 10^{-9} \mathrm{~s}$. However, considering the higher harmonic waves generated by the interactions between the excitation signals and micro-cracks, the time increment is set to be $\Delta t=2 \times 10^{-10} \mathrm{~s}$, to be on the safe side. In addition, a double precision operation is used to reduce the accumulative error here.

Moreover, an additional consideration is the randomness of the crack distribution. To capture the stochastic nature of the random distribution, multiple FEM models are constructed with different realizations of the random distribution. Figure 3 shows the average acoustic nonlinearity parameter versus the number of FEM models ( $\mathrm{U} 1$ and $\mathrm{U} 2$ are the displacements in the $x$ and $y$ direction, respectively), where $c$ is the crack density, $f$ is the excitation frequency, $\mu$ is the friction coefficient of the micro-crack surfaces, and $w \%$ is the proportion of surface cracks. The acoustic nonlinearity parameter is more stable when the number of FEM models is larger than 50. Therefore, the results shown in the following section are averaged over 100 FEM models. 


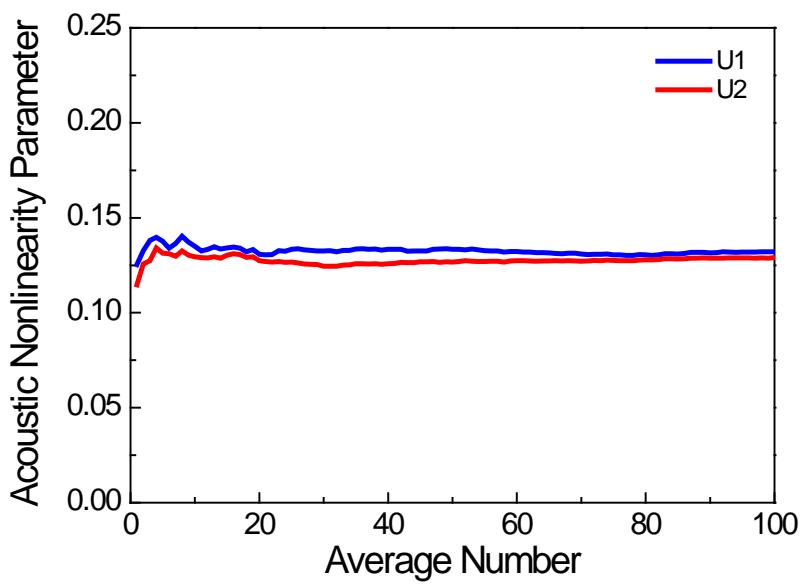

Figure 3. Acoustic nonlinearity parameter versus number of FEM models $(c=0.1, f=1000 \mathrm{kHz}, \mu=0.1$, $w \%=60 \%)$.

\section{Result and Discussion}

When Rayleigh surface waves pass through micro-cracks, they can make the crack faces open under tension and closed under compression, which generates acoustic nonlinearity [24,29]. Meanwhile, because the wavelength $\lambda(1.4-3.6 \mathrm{~mm})$ of Rayleigh surface waves is much larger than the crack length (50 $\mu \mathrm{m}$ to $150 \mu \mathrm{m}$ ), most of the waves can pass through the micro-crack zone. Furthermore, to investigate quantitative relationships between the acoustic nonlinear parameter and the micro-cracks, some representative characteristics or parameters, i.e., the crack density, the propagation distance in the micro-crack zone, the proportion of surface cracks (i.e., $w \%$ ), the frequency of the fundamental wave, and the friction coefficient of the micro-crack surfaces, are considered here.

Figure 4 shows the displacement contour of Rayleigh surface waves while propagating in a structure with surface micro-cracks at $1000 \mathrm{kHz}$. We can see clearly that the wavelength of Rayleigh surface waves is much larger than the crack length. Moreover, although the waves interact with the micro-crack surfaces in the micro-crack zone, no obvious change is observed in the waveforms. Only a very weak scattering phenomenon occurred around the micro-cracks. Meanwhile, the 800 times zoomed deformed shapes clearly demonstrate the opening and closing states of the micro-cracks.

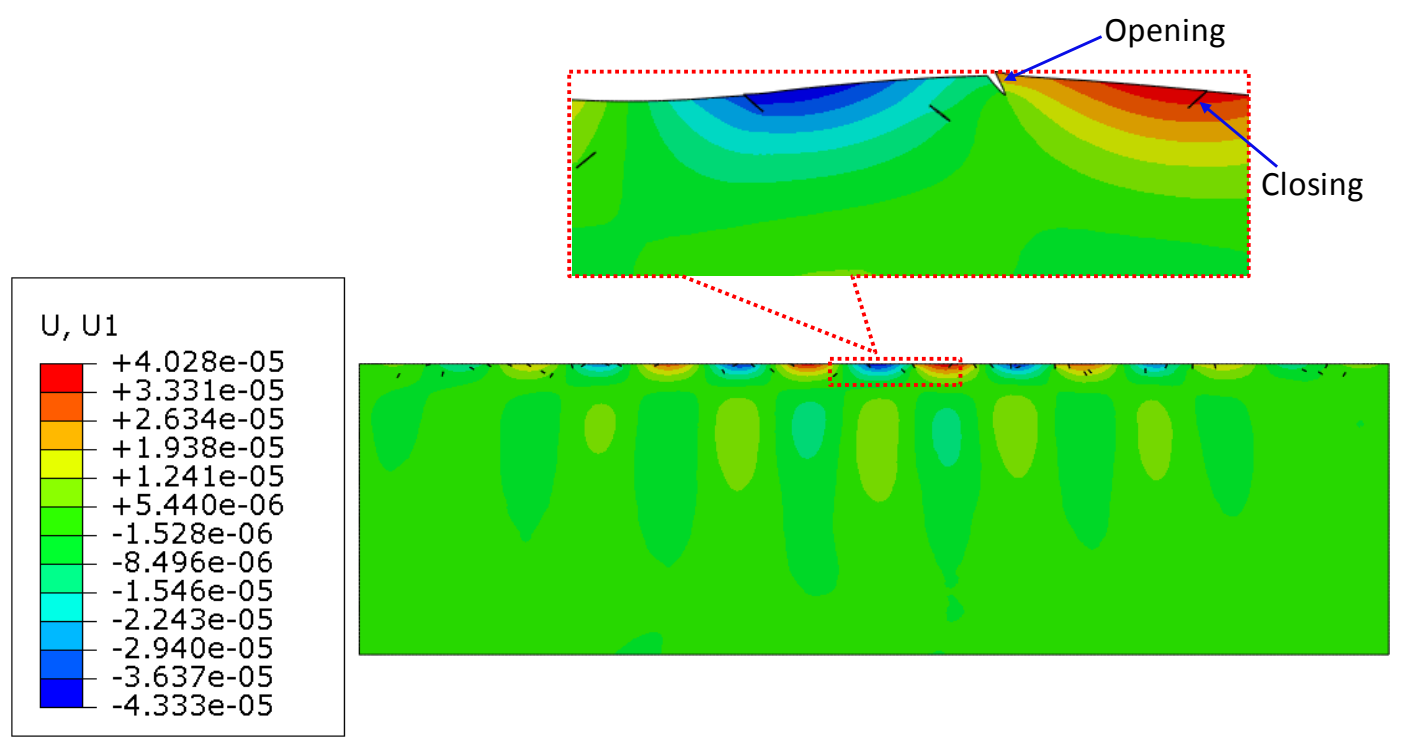

(a)

Figure 4. Cont. 

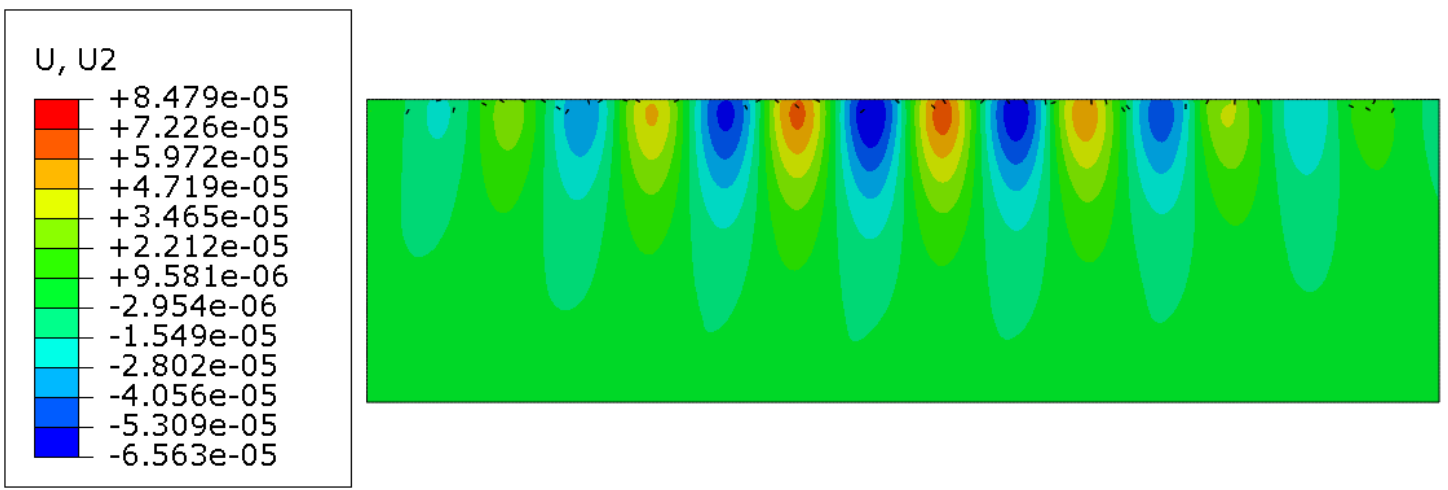

(b)

Figure 4. Propagation of surface waves in a structure with surface micro-cracks $(c=0.1, f=1000 \mathrm{kHz}$, $\mu=0.1, w \%=60 \%$ ). (a) Displacement contour in the $x$ direction (800 times zoomed deformed shapes in the dashed box); (b) Displacement contour in the $y$ direction.

Figures 5 and 6 show wave signals collected at the signal detection position $(0 \mathrm{~mm}$ away from the right boundary of the micro-crack zone) in the $x$ and $y$ directions, respectively, wherein the wave signals for the non-cracked case are marked by the solid line, and the wave signals for the cracked case are marked by the dashed line. We can see that the energies of the wave signals in the $x$ and $y$ directions are almost identical. Moreover, the time-domain signals for the non-cracked case and the cracked case in the $x$ and $y$ directions are very similar, as shown in Figures 5a and 6a, respectively. More obvious differences can be observed from the frequency analysis of the wave signals (Figures $5 b$ and $6 b$ ). We find that the wave signals for the non-cracked case marked by the solid line only contain the $1000 \mathrm{kHz}$ wave signals (the fundamental waves), indicating that the second harmonic waves cannot be generated for the non-cracked case. However, the wave signals for the cracked case, marked by the dashed line, contain not only the $1000 \mathrm{kHz}$ wave signals, but also the $2000 \mathrm{kHz}$ wave signals (second harmonic waves) and even distinct $3000 / 4000 \mathrm{kHz}$ wave signals (third/quadruple harmonic waves), implying that the existence of micro-cracks is the critical factor for generating the second harmonic waves. Meanwhile, the third and quadruple harmonic waves for the case of Rayleigh surface waves are more remarkable compared to the cases of bulk [24] and Lamb [8] waves. Here, from numerical simulations, we find that, when using Rayleigh surface waves, micro-cracks could generate the second harmonic waves. Therefore, this study provides a foundation for some possible new techniques for identifying surface micro-cracks in a structure using Rayleigh surface waves.

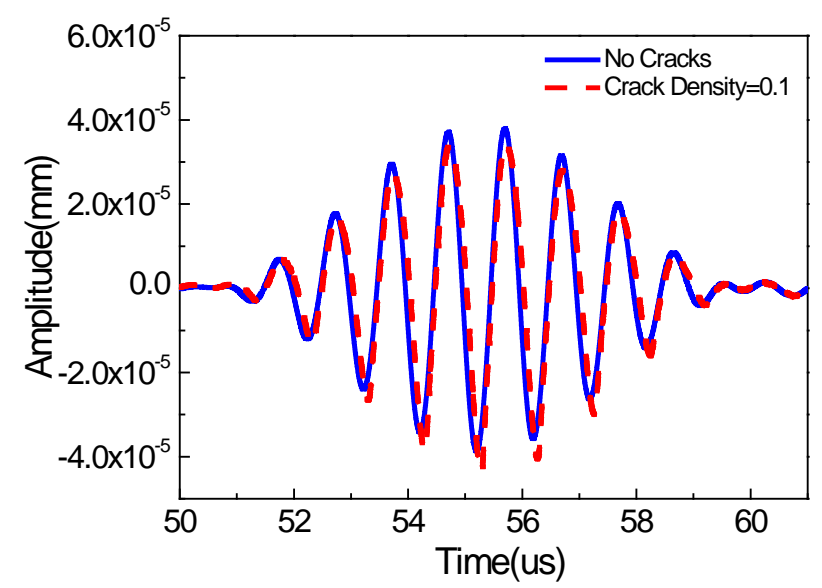

(a)

Figure 5. Cont. 


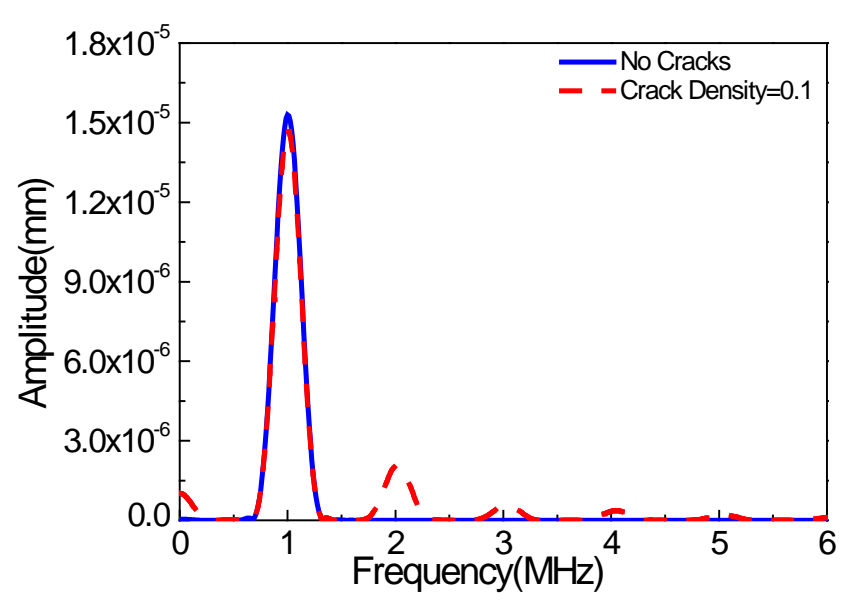

(b)

Figure 5. Wave signals in the $x$ direction (a) time domain; (b) frequency domain $(c=0.1, f=1000 \mathrm{kHz}$, $\mu=0.1, w \%=60 \%)$.

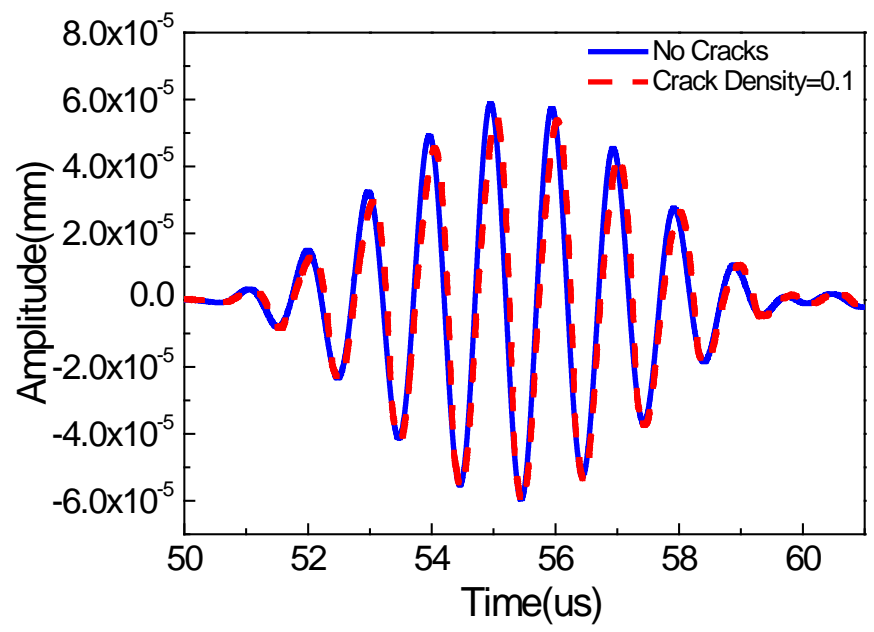

(a)

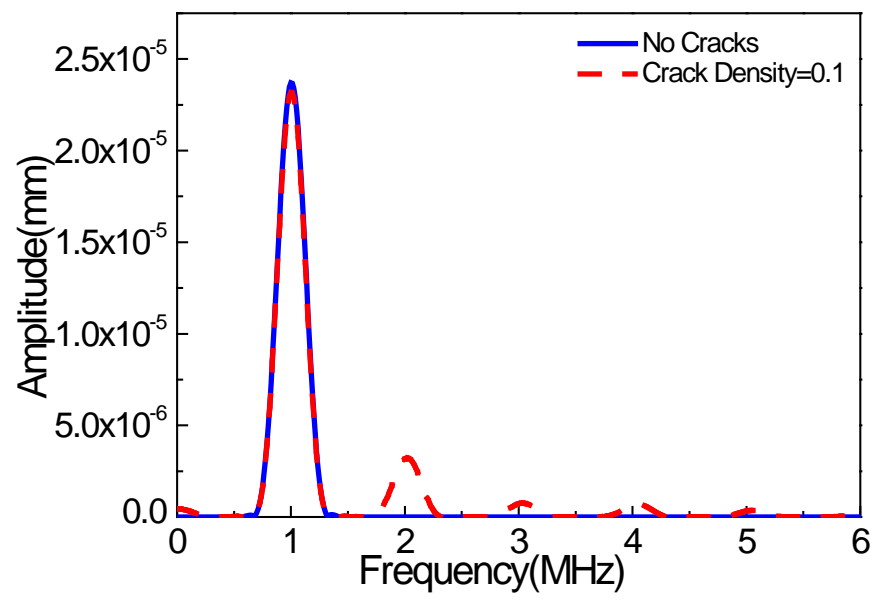

(b)

Figure 6. Wave signals in the $y$ direction (a) time domain; (b) frequency domain $(c=0.1, f=1000 \mathrm{kHz}$, $\mu=0.1, w \%=60 \%)$. 
Next, the wave signals collected at $0 \mathrm{~mm}, 40 \mathrm{~mm}$, and $80 \mathrm{~mm}$, away from the right boundary of the micro-crack zone, are shown in Figure 7 (in the $y$ direction). We can find that the waveforms of Rayleigh surface waves collected at different locations are the same, indicating that the second harmonics from the received signal are independent from the signal detection position. And the same tendency can be found in the $x$ direction. If there exists damping, the amplitudes of the fundamental waves and the second harmonics could be reduced, but nevertheless, the acoustic nonlinear phenomenon cannot be affected significantly.

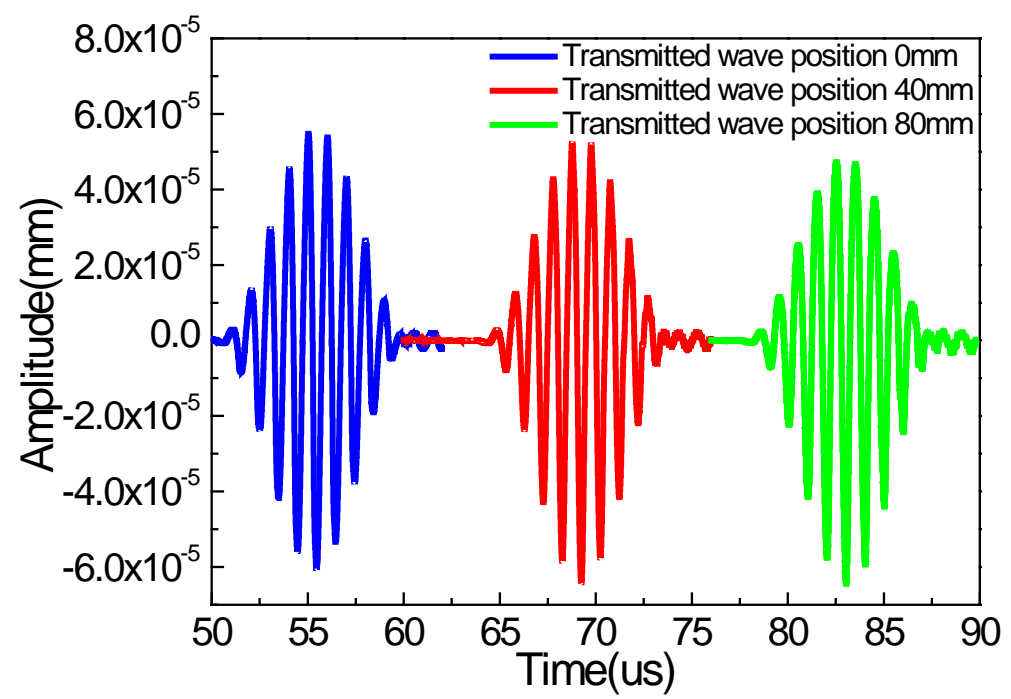

(a)

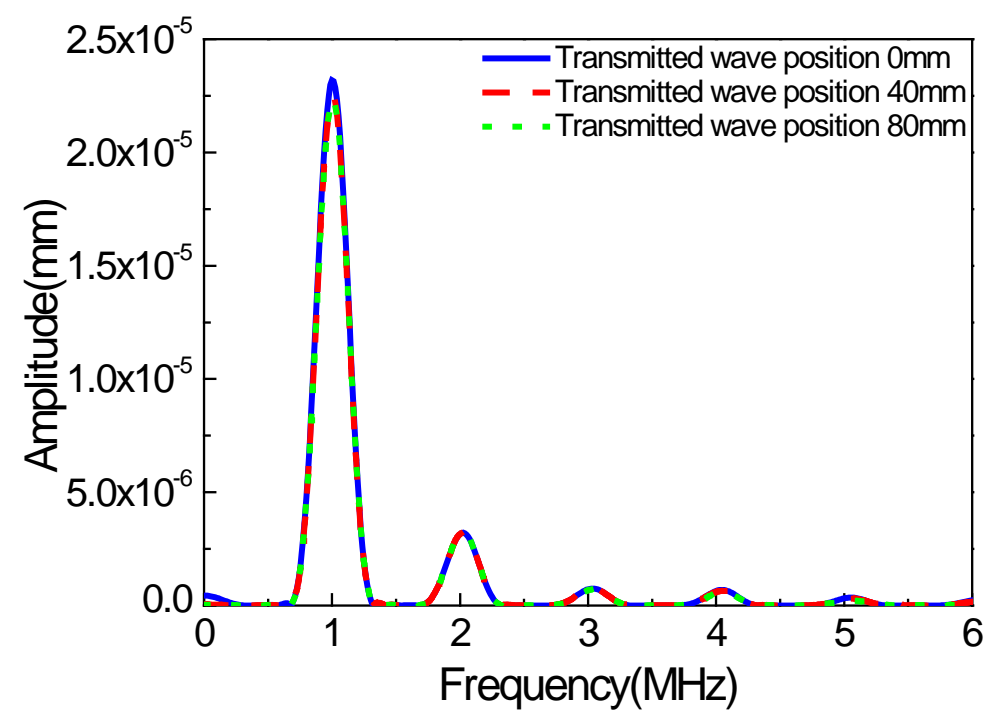

(b)

Figure 7. Waveforms of wave signals in the $y$ direction at different locations (a) time domain; (b) frequency domain $(c=0.1, f=1000 \mathrm{kHz}, \mu=0.1, w \%=60 \%)$.

Furthermore, we investigate the relationship between the acoustic nonlinearity parameter and the proportion of surface micro-cracks, as well as the crack density and propagation distance in the micro-crack zone. The acoustic nonlinearity parameter increases almost linearly with the proportion of surface micro-cracks as shown in Figure 8. When all the micro-cracks are on the surface $(w \%=100 \%)$, the acoustic nonlinearity parameter reaches the maximum, which is the most serious case in practice. 
Figure 9 shows the acoustic nonlinearity parameter versus the crack density $c$. The crack density $(c=N a / L)$ is a dimensionless number, which can be used as a quantitative indicator for acoustic nonlinearity caused by micro-cracks. Because the crack density can be affected by parameters $N$ and $a$, we consider the following two cases: the first case is to increase $N$ and to keep $a$ the same, and the second is to increase $a$ and to keep $N$ the same. When the excitation frequency and the friction coefficient of the micro-crack surfaces remain unchanged, the acoustic nonlinearity parameter is more sensitive to crack length than crack number, and the acoustic nonlinearity parameter increases linearly with the increase in crack density. We also find that the acoustic nonlinearity parameters in the $x$ and $y$ directions are consistent.

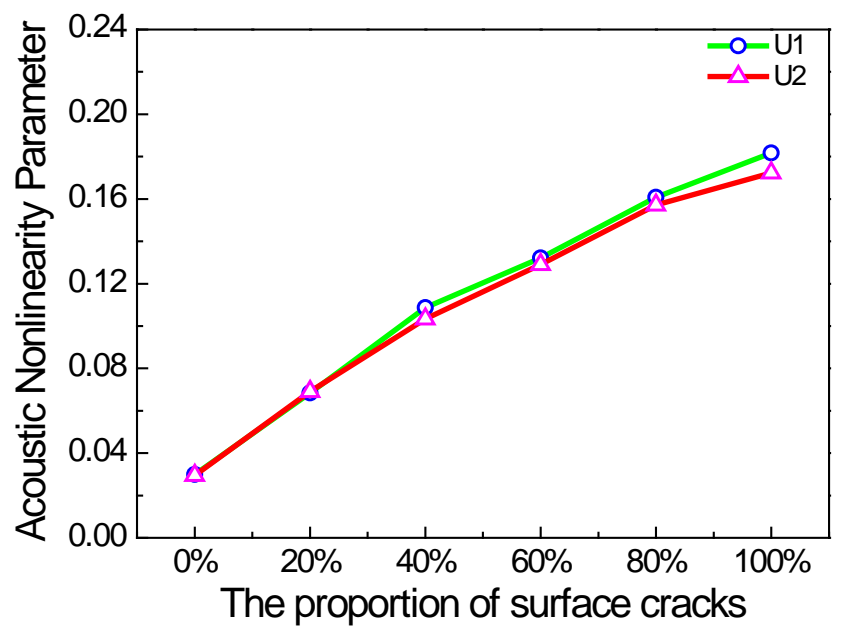

Figure 8. Acoustic nonlinearity parameter versus the proportion of surface cracks $(c=0.1, f=1000 \mathrm{kHz}$, $\mu=0.1)$.

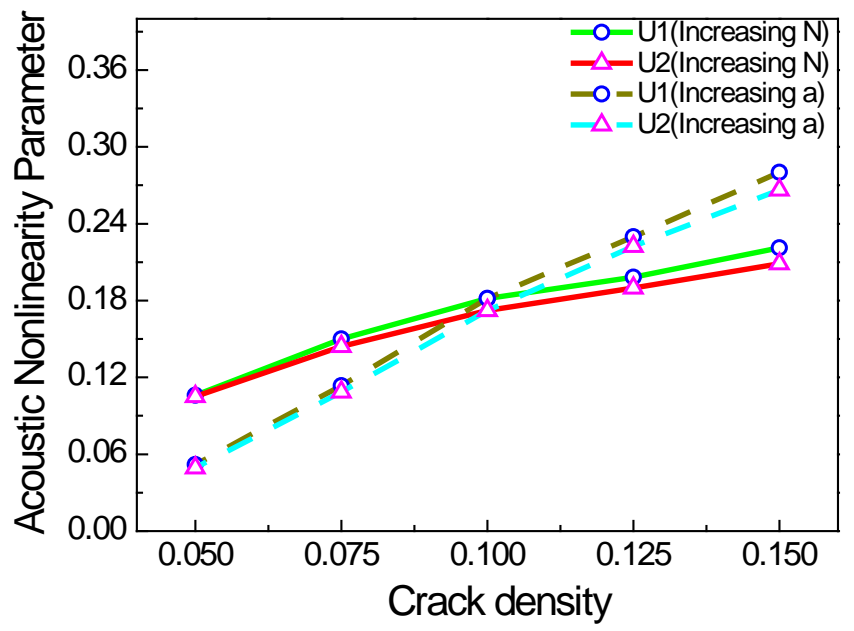

Figure 9. Acoustic nonlinearity parameter versus the crack density $(f=1000 \mathrm{kHz}, \mu=0.1, w \%=100 \%)$.

The lengths of each micro-crack in practice should be different. Thus, we also consider the two cases with the crack length of uniform and Gaussian random distributions. In the two cases, the mean values are the same, but the standard deviations are changed. Figures 10 and 11 show the acoustic nonlinearity parameter versus the standard deviation of micro-crack lengths. It is shown that the acoustic nonlinearity parameter does not change with standard deviation, which is only related to the mean value.

Figure 12 shows the acoustic nonlinearity parameter versus the propagation distance in the micro-crack zone. The acoustic nonlinearity parameter also increases linearly with the propagation distance in the micro-crack zone, leading to its linear accumulation property. 


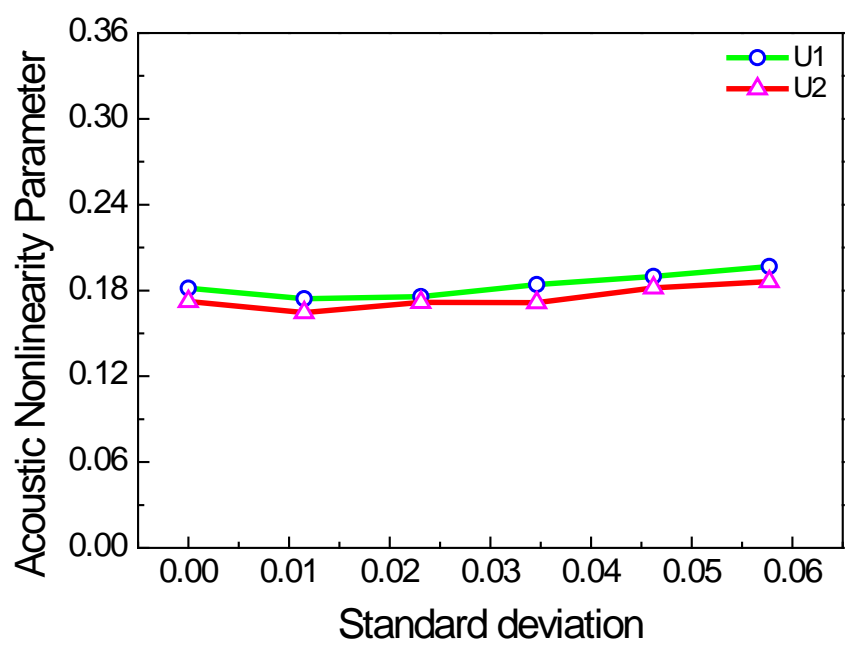

Figure 10. Acoustic nonlinearity parameter versus standard deviation of micro-crack lengths with uniform distribution $(c=0.1, f=1000 \mathrm{kHz}, \mu=0.1, w \%=100 \%)$.

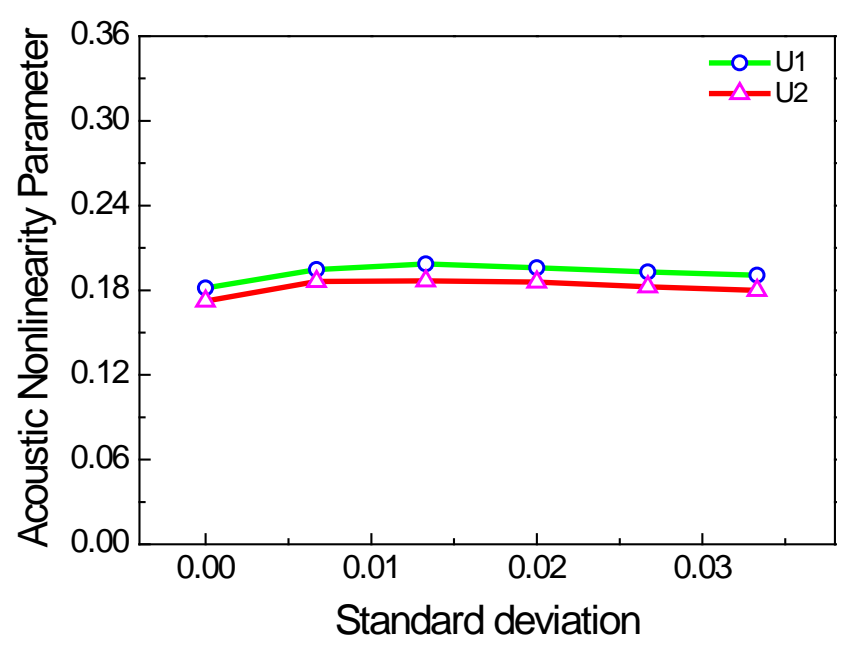

Figure 11. Acoustic nonlinearity parameter versus standard deviation of micro-crack lengths with Gaussian distribution $(c=0.1, f=1000 \mathrm{kHz}, \mu=0.1, w \%=100 \%)$.

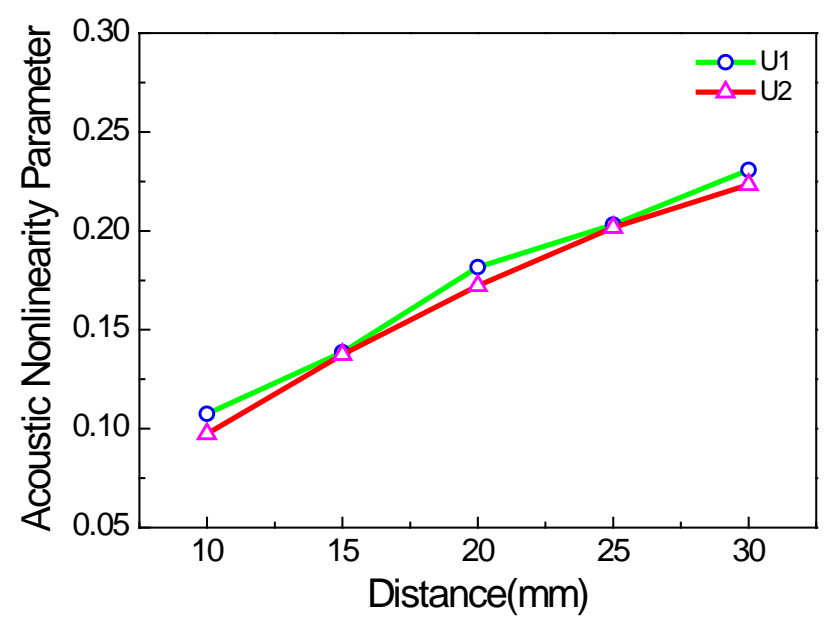

Figure 12. Acoustic nonlinearity parameter versus the propagation distance in the micro-crack zone $(c=0.1, f=1000 \mathrm{kHz}, \mu=0.1, w \%=100 \%)$. 
In contrast to classic acoustic nonlinearity, the factors affecting acoustic nonlinearity also include excitation frequency and the friction coefficient of micro-crack surfaces. In this study, the Coulomb law of friction is used to calculate the frictional force between the micro-crack surfaces. When the crack density and the excitation frequency remain unchanged, the acoustic nonlinearity parameter has almost no relation to the friction coefficient, as shown in Figure 13. It should be noted that the acoustic nonlinearity parameter of the $x$ direction is slightly higher than that of the $y$ direction. The reasons could be the more pronounced clapping behavior of the cracks than the frictional one, and asymmetry due to the random micro-cracks modelling. Thus, it could be inferred that the clapping behavior of the cracks is more remarkable than the frictional one under the condition of randomly distributed micro-cracks. Figure 14 shows the acoustic nonlinearity parameter versus the excitation frequency. It can be seen that the acoustic nonlinearity parameters of the $x$ and $y$ directions increase linearly with the excitation frequency. The main reason is that the wavelength of the Rayleigh surface wave decreases when the excitation frequency increases, and the wave with a smaller wavelength is more sensitive to the micro-crack. Therefore, the nonlinear interactions between Rayleigh surface waves with a smaller wavelength and the micro-cracks became stronger, and the second harmonic was more noticeable.

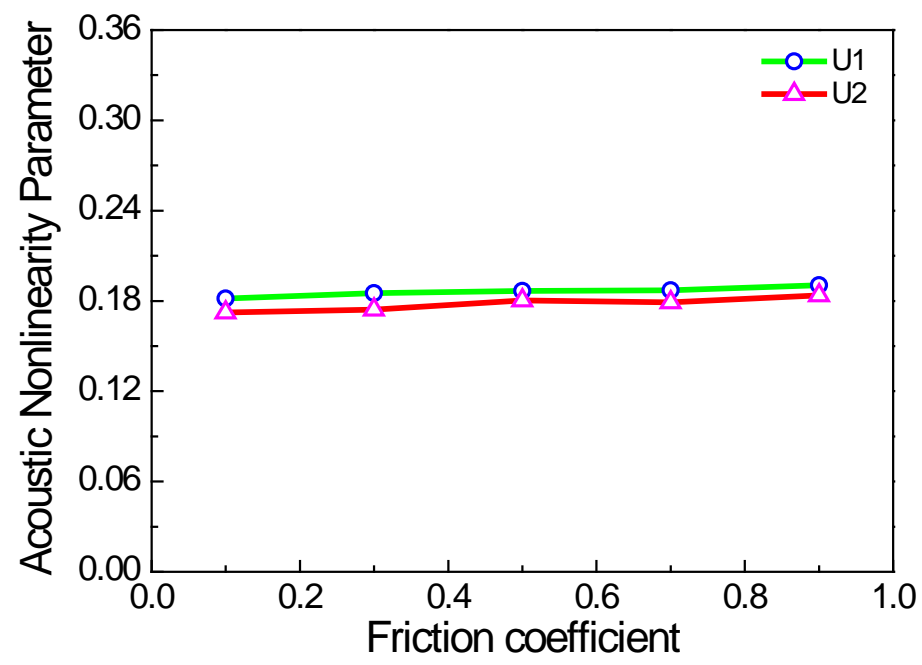

Figure 13. Acoustic nonlinearity parameter versus the friction coefficient $(c=0.1, f=1000 \mathrm{kHz}$, $w \%=100 \%)$.

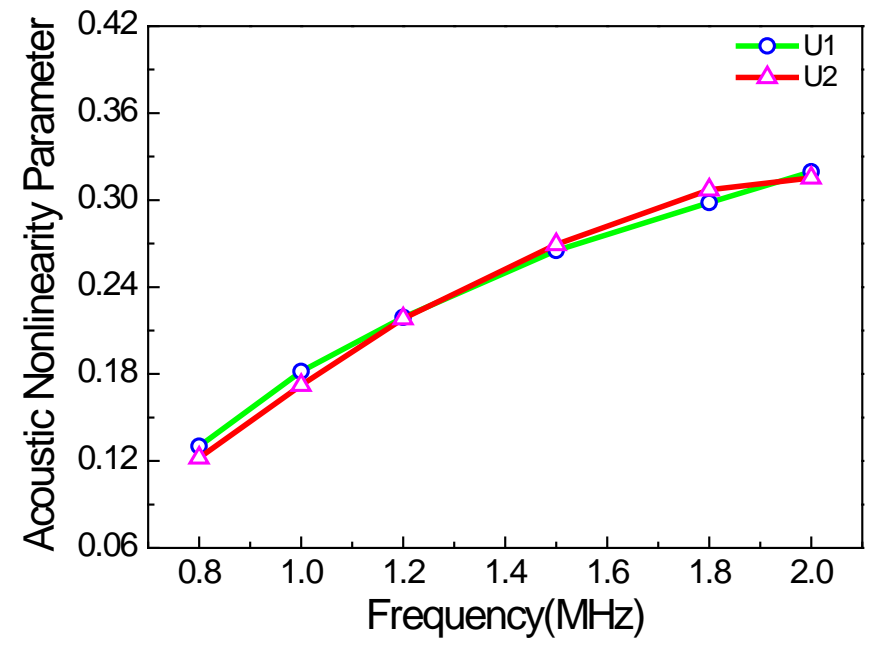

Figure 14. Acoustic nonlinearity parameter versus the excitation frequency $(c=0.1, \mu=0.1, w \%=100 \%)$. 
The above cases are all based on the assumption that crack angles have a uniform and random distribution with a range from $0^{\circ}$ to $180^{\circ}$ (the angle between the crack face and the $x$ direction). However, the angles of micro-cracks $[22,23]$ can affect the acoustic nonlinearity parameter. In this case, we consider that all the micro-crack angles are uniform, and the positions of micro-cracks are also of a uniformly random distribution. The acoustic nonlinearity parameter is shown as a function of the angle of micro-cracks in Figure 15. When the angle is $90^{\circ}$, the acoustic nonlinearity parameter is at the maximum, which is essentially caused by the considerable clapping behavior of the cracks rather than the frictional one. Meanwhile, we also find that the friction coefficient slightly affects the acoustic nonlinearity parameter under the condition of regularly arranged micro-cracks. The reason could be the weak energies of Rayleigh waves, which hardly generate the slipping trend between the surfaces of micro-cracks. Therefore, we can also infer that the clapping behavior is of more importance than the frictional one under the condition of regularly arranged micro-cracks.

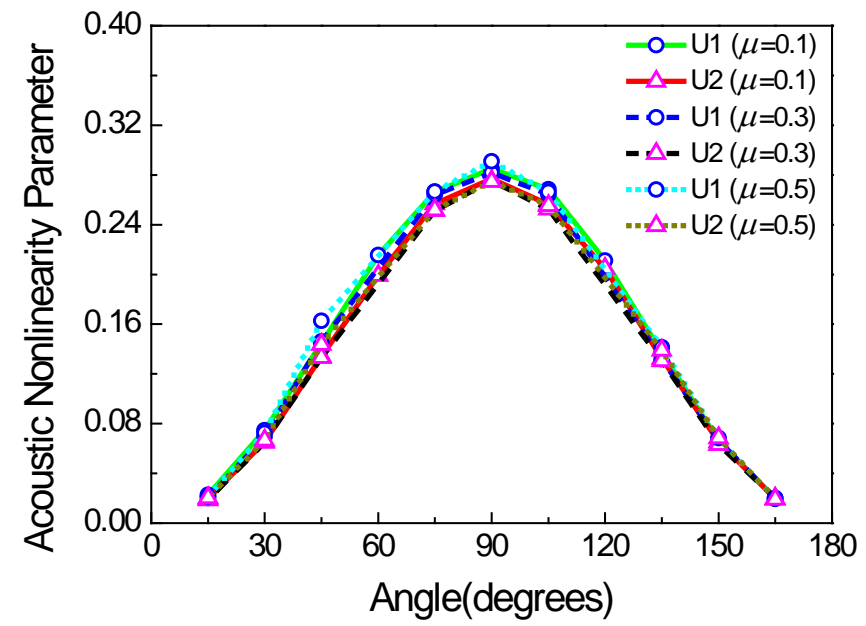

Figure 15. Acoustic nonlinearity parameter versus the angle of micro-cracks $(c=0.1, f=1000 \mathrm{kHz}$, $w \%=100 \%)$.

\section{Conclusions}

A numerical model containing randomly distributed surface micro-cracks is constructed to investigate the propagation phenomenon of Rayleigh surface waves by statistical analysis from numerous results from random micro-crack models. The following paragraphs outline the main conclusions drawn from this study.

Firstly, when Rayleigh surface waves are used as the fundamental waves, the ultrasonic nonlinear effect, in terms of second harmonics, can be generated by surface micro-cracks. It is found that the third and quadruple harmonics of Rayleigh surface waves are more noteworthy than those of bulk and Lamb waves.

Second, the different distributions of the lengths and angles of surface micro-cracks were investigated. The results show that the acoustic nonlinearity parameter is only related to the mean value of the lengths of surface micro-cracks, and the acoustic nonlinearity parameter is at maximum when the angle of the surface micro-crack is $90^{\circ}$.

Finally, we systematically investigated the relationships between the acoustic nonlinear parameter and some key factors, i.e., the micro-crack density, the propagation distance in the cracking region, the proportion of surface cracks, the excitation frequency, and the friction coefficient of the micro-crack surfaces. The results reveal that the acoustic nonlinear parameters in the $x$-axis and $y$-axis are basically consistent, which is linearly proportional to the crack density, the proportion of surface cracks, the propagation distance of surface waves in the cracking region, and the excitation frequency. However, it is not correlated with the friction coefficient of the micro-crack surfaces. Therefore, 
the acoustic nonlinear parameter can be used to effectively characterize the degeneration of material properties caused by surface micro-cracks using the Rayleigh surface wave technique. Furthermore, a proper increase of the fundamental frequency can generate more significantly higher harmonics and improve the accuracy of this technique. These quantitative relationships provide the foundation for developing nonlinear ultrasonic based quantitative non-destructive evaluation techniques for assessing surface micro-cracks.

Acknowledgments: This work is supported by the National Science Foundation of China (No. 11604033, No. 11632004, No. 51769028 and No. 11602040), and Key Program for International Science and Technology Cooperation Projects of Ministry of Science and Technology of China (No. 2016YFE0125900), Key Project of Natural Science Foundation of CQ CSTC (No. 0241002432002).

Author Contributions: X.D., F.L., Y.X., P.C. and M.D. conceived and designed the simulations; F.L. performed the simulations; X.D. analyzed the data; N.H. contributed analysis tools; Y.Z., N.H. and P.C. revised the paper; Y.Z. and N.H. wrote the paper.

Conflicts of Interest: The authors declare no conflict of interest.

\section{References}

1. Sposito, G.; Ward, C.; Cawley, P.; Nagy, P.B.; Scruby, C. A review of non-destructive techniques for the detection of creep damage in power plant steels. NDT E Int. 2010, 43, 555-567. [CrossRef]

2. Qiu, J.X.; Pei, C.X.; Liu, H.C.; Chen, Z.M.; Demachi, K. Remote inspection of surface cracks in metallic structures with fiber-guided laser array spots thermography. NDT E Int. 2017, 92, 213-220. [CrossRef]

3. Cantrell, J.H.; Yost, W.T. Nonlinear ultrasonic characterization of fatigue microstructures. Int. J. Fatigue 2001, 23, 487-490. [CrossRef]

4. Ogi, H.; Hirao, M.; Aoki, S. Noncontact monitoring of surface-wave nonlinearity for predicting the remaining life of fatigued steels. J. Appl. Phys. 2001, 90, 438-442. [CrossRef]

5. Lee, T.H.; Jhang, K.Y. Experimental investigation of nonlinear acoustic effect at crack. NDT E Int. 2009, 42, 757-764. [CrossRef]

6. Oberhardt, T.; Kim, J.-Y.; Qu, J.; Jacobs, L.J. A contact mechanics based model for partially-closed randomly distributed surface microcracks and their effect on acoustic nonlinearity in Rayleigh surface waves. AIP Conf. Proc. 2016, 1706, 020024.

7. Wan, X.; Tse, P.W.; Xu, G.; Tao, T.; Liu, F.; Chen, X.; Zhang, Q. Fem simulation of nonlinear lamb waves for detecting a micro-crack in a metallic plate. In Engineering Asset Management-Systems, Professional Practices and Certification; Springer: Cham, Switzerland, 2015; pp. 1561-1569.

8. Zhao, Y.X.; Li, F.L.; Cao, P.; Liu, Y.L.; Zhang, J.Y.; Fu, S.Y.; Zhang, J.; Hu, N. Generation mechanism of nonlinear ultrasonic Lamb waves in thin plates with randomly distributed micro-cracks. Ultrasonics 2017, 79, 60-67. [CrossRef] [PubMed]

9. Deng, M.; Xiang, Y.; Liu, L. Time-domain analysis and experimental examination of cumulative second-harmonic generation by primary lamb wave propagation. J. Appl. Phys. 2011, 109, 113525. [CrossRef]

10. Hong, M.; Su, Z.; Wang, Q.; Cheng, L.; Qing, X. Modeling nonlinearities of ultrasonic waves for fatigue damage characterization: Theory, simulation, and experimental validation. Ultrasonics 2014, 54, 770-778. [CrossRef] [PubMed]

11. Shen, Y.; Giurgiutiu, V. Predictive modeling of nonlinear wave propagation for structural health monitoring with piezoelectric wafer active sensors. J. Intell. Mater. Syst. Struct. 2014, 25, 506-520. [CrossRef]

12. Rauter, N.; Lammering, R. Investigation of the higher harmonic lamb wave generation in hyperelastic isotropic material. Phys. Procedia 2015, 70, 309-313. [CrossRef]

13. Achenbach, J.D.; Wang, Y. Far-field resonant third harmonic surface wave on a half-space of incompressible material of cubic nonlinearity. J. Mech. Phys. Solids 2017. [CrossRef]

14. Herrmann, J.; Kim, J.Y.; Jacobs, L.J.; Qu, J.; Littles, J.W.; Savage, M.F. Assessment of material damage in a nickel-base superalloy using nonlinear Rayleigh surface waves. J. Appl. Phys. 2006, 99, 1479-1488. [CrossRef]

15. Thiele, S.; Kim, J.Y.; Qu, J.; Jacobs, L.J. Air-coupled detection of nonlinear Rayleigh surface waves to assess material nonlinearity. Ultrasonics 2014, 54, 1470-1475. [CrossRef] [PubMed] 
16. Torello, D.; Thiele, S.; Matlack, K.H.; Kim, J.Y.; Qu, J.; Jacobs, L.J. Diffraction, attenuation, and source corrections for nonlinear Rayleigh wave ultrasonic measurements. Ultrasonics 2015, 56, 417-426. [CrossRef] [PubMed]

17. Walker, S.V.; Kim, J.Y.; Qu, J.; Jacobs, L.J. Fatigue damage evaluation in A36 steel using nonlinear Rayleigh surface waves. NDT E Int. 2012, 48, 10-15. [CrossRef]

18. Zabolotskaya, E.A. Nonlinear propagation of plane and circular Rayleigh waves in isotropic solids. J. Acoust. Soc. Am. 1992, 91, 2569-2575. [CrossRef]

19. Tiersten, H.F.; Baumhauer, J.C. Second harmonic generation and parametric excitation of surface waves in elastic and piezoelectric solids. J. Appl. Phys. 1980, 45, 4272-4287. [CrossRef]

20. Zabolotskaya, E.A.; Ilinskii, Y.A.; Hamilton, M.F. Nonlinear surface waves in soft, weakly compressible elastic media. J. Acoust. Soc. Am. 2007, 121, 1873-1878. [CrossRef] [PubMed]

21. Zeitvogel, D.T.; Matlack, K.H.; Kim, J.Y.; Jacobs, L.J.; Singh, P.M.; Qu, J. Characterization of stress corrosion cracking in carbon steel using nonlinear Rayleigh surface waves. NDT E Int. 2014, 62, 144-152. [CrossRef]

22. Zhao, Y.; Qiu, Y.; Jacobs, L.J.; Qu, J. Frequency-dependent tensile and compressive effective moduli of elastic solids with randomly distributed two-dimensional microcracks. J. Appl. Mech. 2015, 82, 081006. [CrossRef]

23. Zhao, Y.; Qiu, Y.; Jacobs, L.J.; Qu, J. Frequency-dependent tensile and compressive effective moduli of elastic solids with distributed penny-shaped microcracks. Acta Mech. 2016, 227, 399-419. [CrossRef]

24. Zhao, Y.; Qiu, Y.; Jacobs, L.J.; Qu, J. A micromechanics model for the acoustic nonlinearity parameter in solids with distributed microcracks. In 42nd Annual Review of Progress in Quantitative Nondestructive Evaluation: Incorporating the 6th European-American Workshop on Reliability of NDE; AIP Publishing: Melville, NY, USA, 2016; p. 060001.

25. Rjelka, M.; Koehler, B.; Mayer, A. Extraction of depth profiles of third-order elastic constants in cracked media. In 43rd Review of Progress in Quantitative Nondestructive Evaluation; American Institute Physics: Melville, NY, USA, 2017; Volume 1806.

26. Matlack, K.H.; Kim, J.Y.; Jacobs, L.J.; Qu, J. Review of second harmonic generation measurement techniques for material state determination in metals. J. Nondestruct. Eval. 2015, 34, 273. [CrossRef]

27. Jiao, J.; Meng, X.; He, C.; Wu, B. Nonlinear lamb wave-mixing technique for micro-crack detection in plates. NDT E Int. 2016, 85, 63-71.

28. Shen, Y.; Cesnik, C.E.S. Modeling of nonlinear interactions between guided waves and fatigue cracks using local interaction simulation approach. Ultrasonics 2016, 74, 106-123. [CrossRef] [PubMed]

29. Solodov, I.Y.; Krohn, N.; Busse, G. Can: An example of nonclassical acoustic nonlinearity in solids. Ultrasonics 2002, 40, 621-625. [CrossRef]

(C) 2018 by the authors. Licensee MDPI, Basel, Switzerland. This article is an open access article distributed under the terms and conditions of the Creative Commons Attribution (CC BY) license (http://creativecommons.org/licenses/by/4.0/). 